\title{
TANDACUCHIS (Ascalapha odorata Linnaeus, 1758. Familia Noctuidae): PERCEPCIONES MÍTICAS Y SOCIALES EN EL CENTRO HISTÓRICO DE QUITO
}

\author{
Tandlacuchis (Ascalapha odorata Linnaeus, 1758. Family Noctuidae): \\ mythical and social perceptions in the historical center of Quito
}

\author{
Karina Paola BUITRÓN - BUITRÓN ${ }^{1 *}$, Kevin Alexander TOAPANTA-AMÁN ${ }^{1}$, Cristina Monserrate \\ NARVÁEZ - VALDIVIESO ${ }^{1}$, Wendy Betzabé PACHECO - PAREDES ${ }^{1}$
}

${ }^{1}$ Universidad Central del Ecuador, Facultad de Ciencias Biológicas, Carrera de Ciencias Biológicas y Ambientales, Yaguachi y Numa Pompillo Llona, Quito, Ecuador.*kpbuitron@uce.edu.ec

Submitted: 23/08/2020; Accepted: 15/10/2020; Published: 10/01/2021

\section{RESUMEN}

La mariposa nocturna, Ascalapha odorata, es una especie perteneciente al Orden Lepidoptera, Familia Noctuidae, cuya presencia y avistamiento se asocia con presagios negativos que han pasado a través de un flujo de información entre generaciones. Es así, que las personas de la tercera edad son quienes han sido esenciales en la difusión de mitos e historias que rodean a esta especie. En el Centro Histórico de Quito existe gran presencia de adultos mayores, quienes se agrupan en esta zona como pasatiempo, lo cual hace que este sitio sea relevante para realizar un estudio sobre las percepciones y conocimientos que las personas tienen sobre esta especie de mariposa nocturna. Para poder ejecutarlo se realizaron 40 entrevistas que incorporaron datos sociales del individuo, así como preguntas relacionadas con la problemática, entre las más relevantes se puede mencionar el conocimiento sobre el nombre común de la especie, la asociación que le dan a la presencia de Ascalapha odorata en sus hogares y las reacciones que estas personas tienen ante la presencia de la especie. Esto resulta relevante para entender el conocimiento mítico que se alberga en torno a la especie. Para esto se ha planteado como objetivo determinar las percepciones y conocimientos sobre "Tandacuchi" (Ascalapha odorata) por parte de adultos mayores de la Plaza Grande en la ciudad de Quito, sector Centro Histórico.

PALABRAS CLAVE: Tandacuchi, percepciones, adulto mayor, centro histórico.

\begin{abstract}
The nocturnal butterfly Ascalapha odorata is a species from the Order Lepidoptera, Family Noctuidae, whose presence and sighting are associated with negative omens that have passed through a flow of information between generations. Thus, older people are the ones who have been essential in the dissemination of myths and stories that surround this species. In the Historic Center of Quito there is a large presence of older adults, who are grouped in this area as a hobby, which makes this site relevant for a study on the perceptions and knowledge that people have about this species of nocturnal butterfly. In order to carry it out, 40 interviews were carried out that incorporated socioeconomic data on the individual, as well as questions related to the problem, among the most relevant we can mention the knowledge about the common name of the species, the association that they give to the presence of Ascalapha odorata in their homes and the reactions that these people have to the presence of the species. Which is relevant to understand the mythical knowledge. For this purpose, the objective has been to determine the perceptions and knowledge about the Tandacuchis (Ascalapha odorata) by older adults in the Plaza Grande in the City of Quito.
\end{abstract}

KEYWORDS: Tandacuchi, perceptions, older adult, historic center. 
BUITRÓN-BUITRÓN et al. Tandacuchis (Ascalopha odorata Linnaeus, 1978. Familia Noctuidai): percepciones míticas y sociales en el centro histórico de Quito. Ethnoscientia, 6, 2021. D.O.I.: 10.22276/ethnoscientia.v6i1.336

\section{INTRODUCCIÓN}

Los estudios etnozoológicos constituyen una fuente valiosa de información, ya que reflejan el simbolismo, conocimiento y valor de los animales desde la cosmovisión andina (WAWRZYK, 2013). Ascalapha odorata es una especie migratoria que se caracteriza por presentar en su cuerpo un dorso café oscuro, franjas blancas y manchas negras en las alas con longitud de $15 \mathrm{~cm}$ de largo (SETH et al., 2017), se distribuye desde Canadá hasta Argentina y además su alimentación consiste del follaje de algunas fabáceas (EKREM et al., 2014).

Bertonatti (2013) afirma que: la "mariposa negra" tiene una percepción de mala suerte en varios países, conociéndola como: "mariposa de la muerte", "bruja negra" (Paraguay), "ura" (Paraguay) o "tarabuja” (Venezuela). Barragán y colaboradores (2009) consideran a Ascalapha odorata como mensajero de la muerte cuando ingresa en los hogares desde tiempos precolombinos. Quinn (2017) observa un descenso poblacional del insecto en México debido a la superstición y temor de la misma en poblaciones humanas.

Las percepciones míticas que las personas atribuyen a esta especie resultan de importancia para su conservación, dado que estos son organismos que en su interacción con los seres humanos desencadenan percepciones y reacciones negativas. Esta especie presenta una función ecológica dentro del paisaje urbano, en el cual es un importante organismo polinizador de plantas con floración nocturna, además de formar parte de la dieta esencial de pequeños mamíferos, aves y murciélagos insectívoros (LÓPEZ, 2017; MARTíNEZ et al., 2006; MONTERO et al., 2013). El presente estudio permitió conocer las percepciones que los adultos mayores del centro histórico de Quito mantienen hacia Ascalapha odorata y las razones por las que su presencia causa conmoción y desagrado en los habitantes. En este contexto, el objetivo del estudio fue determinar las percepciones y conocimientos sobre el Tandacuchi (Ascalapha odorata) por parte de adultos mayores de la Plaza Grande en la ciudad de Quito, sector Centro Histórico. El trabajo es realizable debido a su bajo costo económico, además de que el sitio de muestreo se encuentra en la centralidad de la urbe, por lo cual existe facilidad para acceder a los entrevistados.

\section{MATERIAL Y MÉTODOS}

La Plaza Grande, también llamada Plaza de la Independencia o casco colonial, se encuentra ubicada en el centro de Quito, provincia de Pichincha - Ecuador, aproximadamente a 2800 msnm y con una extensión de 8100 metros cuadrados (Fig. 1). El casco colonial cuenta con edificaciones patrimoniales, las cuales lo han hecho acreedor a 
ser considerado como Patrimonio Cultural de la Humanidad por la UNESCO (RAMÍREZ, 2016). Su composición florística incluye árboles patrimoniales y especies nativas y no nativas como: magnolia Magnolia grandiflora, porotón Erythrina edulis, palma de cera Ceroxylon ventricosum, palma australiana Livistona australis, palma abanico Trachycarpus fortunei, araucaria Araucaria angustifolia, higo Ficus carica y arrayán Myrcianthes hallii, además de contar con un gran número de especies de geranios de origen africano Pelargonium spp (CUEVA, 2010). En cuanto a la fauna del sitio, se presenta una dominancia por distintas especies de aves, siendo las más comunes la tórtola orejuda Zenaida auriculata, la paloma Columba livia, el gorrión criollo Zonotrichia capensis y el mirlo Turdus fuscater (CUVI, 2017).

Para la obtención de información acerca del conocimiento etnozoológico de los transeúntes adultos mayores de la Plaza Grande Quito (Fig. 1.) se aplicó una encuesta cualitativa (GARCÍA, et al., 2017) en el periodo de una semana del mes de mayo en el año 2019 (Fig. 3). La encuesta estuvo compuesta por imágenes ilustrativas (Fig. 2) y 8 preguntas estructuradas acerca de las percepciones y conocimientos que las personas tienen acerca de esta especie.
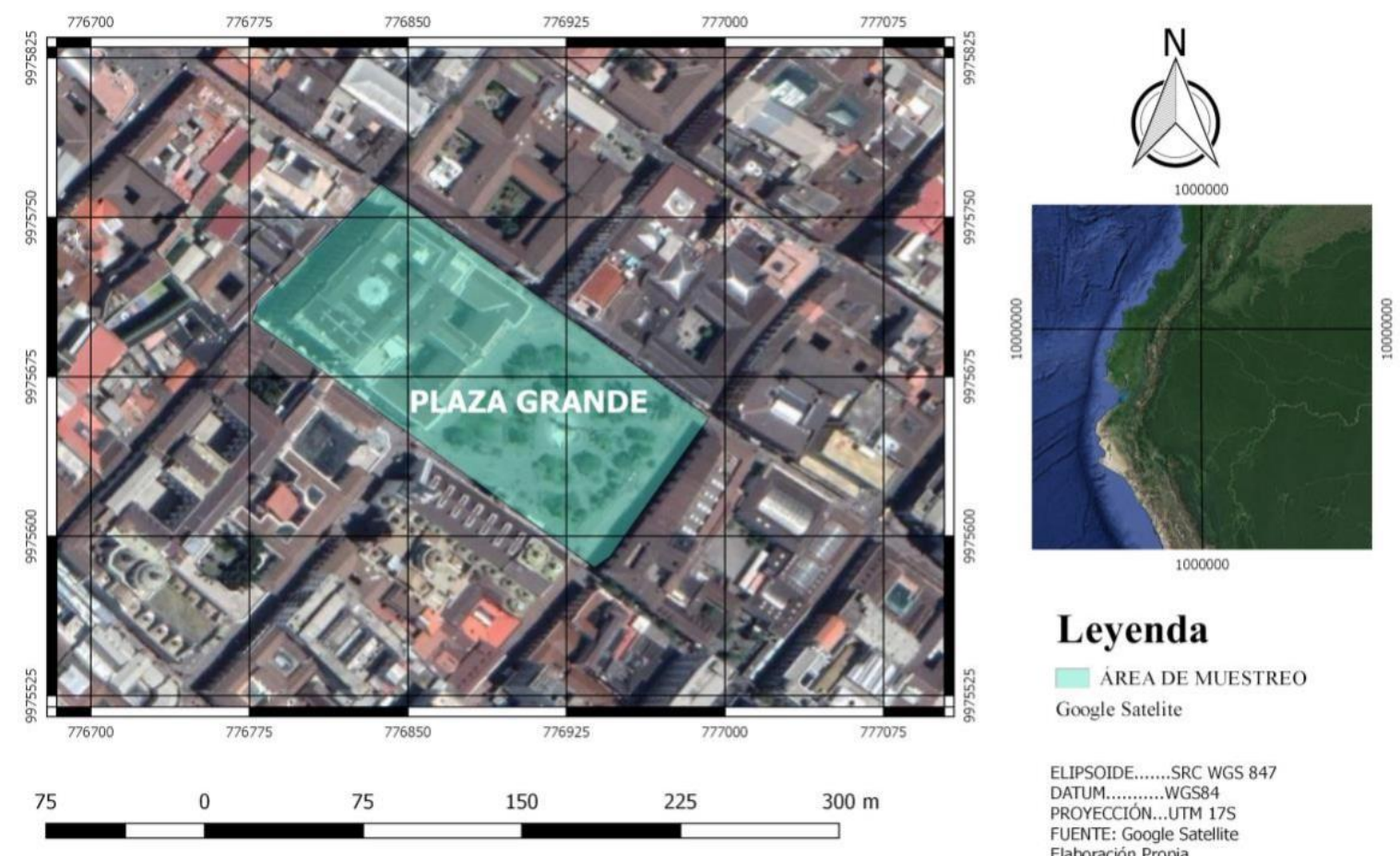

\section{Leyenda}

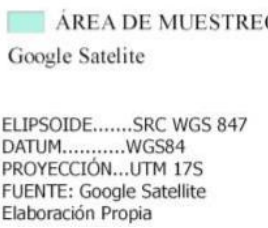

Figura 1: Área de muestreo- Centro Histórico del Distrito Metropolitano de Quito. Fuente: Buitrón y Toapanta (2019). 
Para este estudio los entrevistados fueron adultos mayores de 50 años en adelante, incluyendo jubilados y ancianos en general, sin exclusión étnica o de género y que además son residentes actuales del centro histórico de Quito y sus alrededores.

En primer lugar, se presentó una fotografía referencial de Ascalapha odorata (Fig.2), de esta forma se logró identificar aquellas personas que presentaban algún conocimiento sobre la especie (MARTÍNEZ, 2010). El total de entrevistados fue de 40 personas a quienes se les aplicó la entrevista, previo su consentimiento y voluntaria participación.

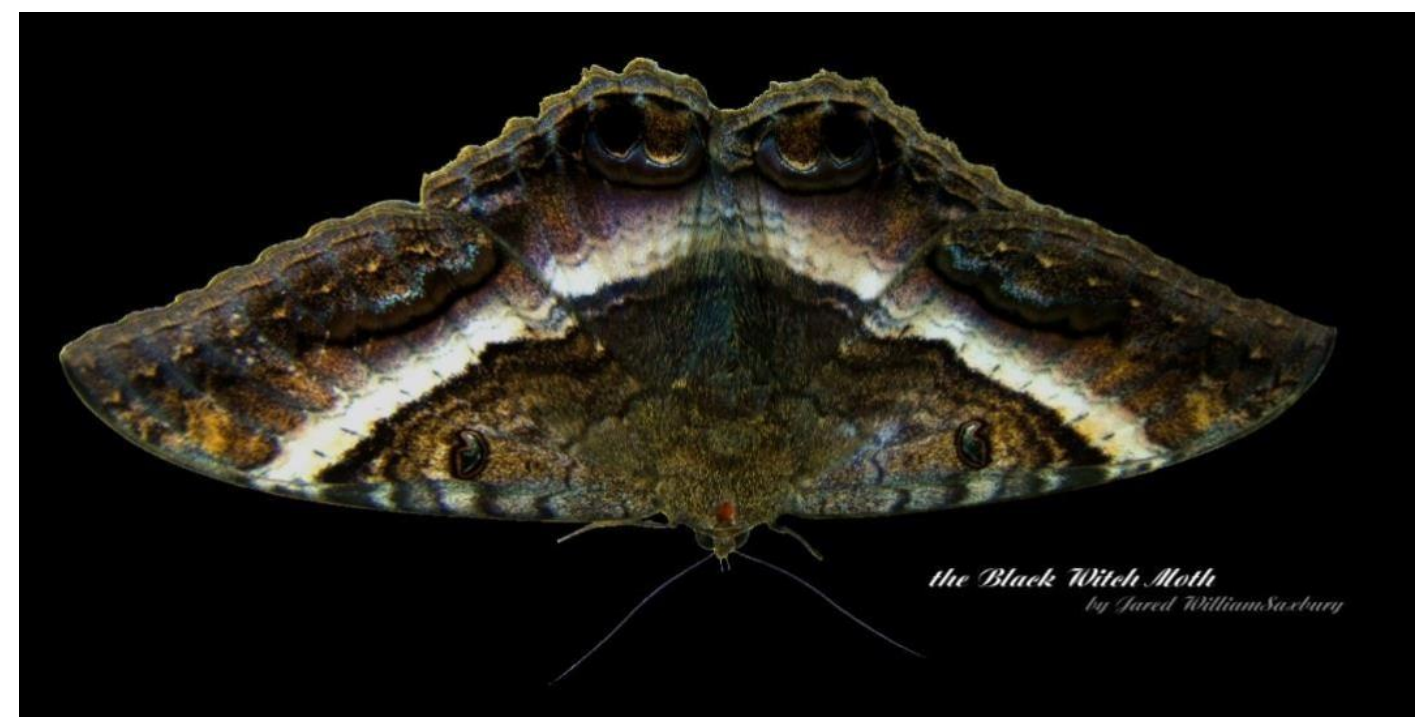

Figura 2: Fotografía referencial de Ascalapha odorata. Fuente: Williams (2011).

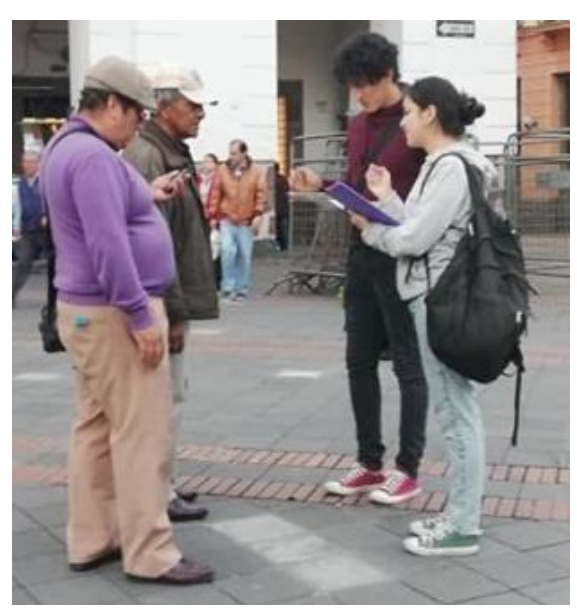

Figura 3: Ejecución de la metodología. Fuente: los autores (2019).

\subsection{Análisis estadístico}

Con los datos cualitativos extraídos a partir de las entrevistas se aplicó estadística descriptiva mediante la herramienta informática Excel para obtener sumatorias totales, así 
BUITRÓN-BUITRÓN et al. Tandacuchis (Ascalopha odorata Linnaeus, 1978. Familia Noctuidai): percepciones míticas y sociales en el centro histórico de Quito. Ethnoscientia, 6, 2021. D.O.I.: $\underline{10.22276 / \text { ethnoscientia.v6i1.336 }}$

como porcentajes. Para visualizar y analizar de mejor manera los resultados se realizaron gráficos de tablas y pasteles porcentuales.

\section{RESULTADOS}

Del total $100 \%$ de entrevistados (40), se presentó un $52.5 \%$ (21) para el género masculino y un $47.5 \%$ (19) correspondientes al género femenino. El 37.5\% (15) de la muestra mencionó conocer a la especie como "Tandacuchi", el 10\% (4) lo reconocen como "Mariposa de la muerte", el 7.5\% (3) lo reconocen como "Mariposa negra", el 7.5\% (3) lo llaman "Mariposa Grande" y para los nombres Mariposa, Chimilaco, Polilla, Payacucha, Mariposa nocturna, Murciélago, Mariposa Café y Robashua se obtuvo un 2.5\% (1) para cada opción (Fig. 4).

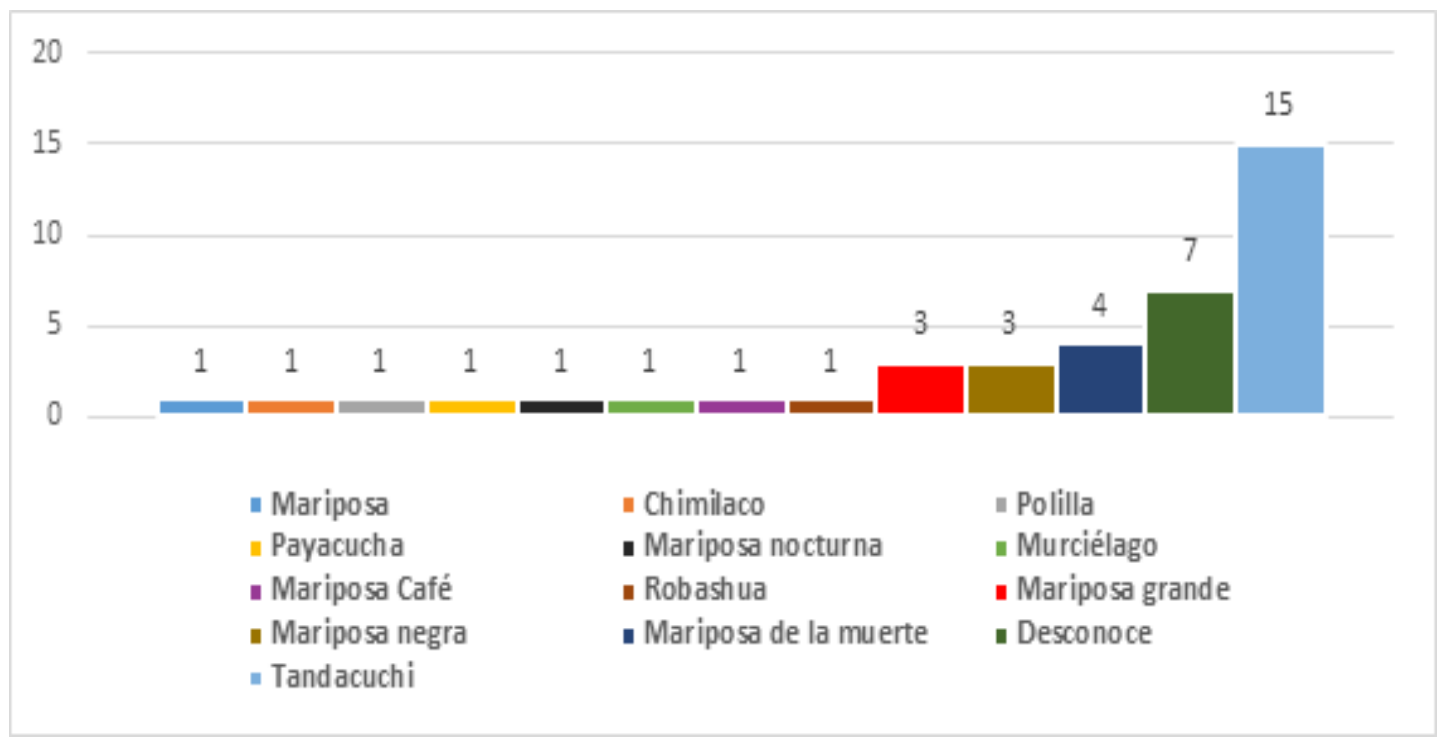

Figura 4: Nombres atribuidos a A. odorata.

El 35\% (14) de los entrevistados asociaron la presencia de esta especie como anunciante de muerte, seguido por un $22.5 \%$ (9) que lo asocian como atrayente de mala suerte, así mismo un $17.5 \%$ (7) la relacionaron como anunciante de robos. Con respecto a la opción "otros" con 3.5\% (6) de los entrevistados sugirieron que la asocian con energía negativa o que es una especie tóxica, además de ser un organismo asociado con entes demoníacos (Fig. 5). El 63\% (25) de los entrevistados mostraron desagrado al observar a la especie directamente (Fig. 6). 
BUITRÓN-BUITRÓN et al. Tandacuchis (Ascalopha odorata Linnaeus, 1978. Familia Noctuidai): percepciones míticas y sociales en el centro histórico de Quito. Ethnoscientia, 6, 2021. D.O.I.: 10.22276/ethnoscientia.v6i1.336

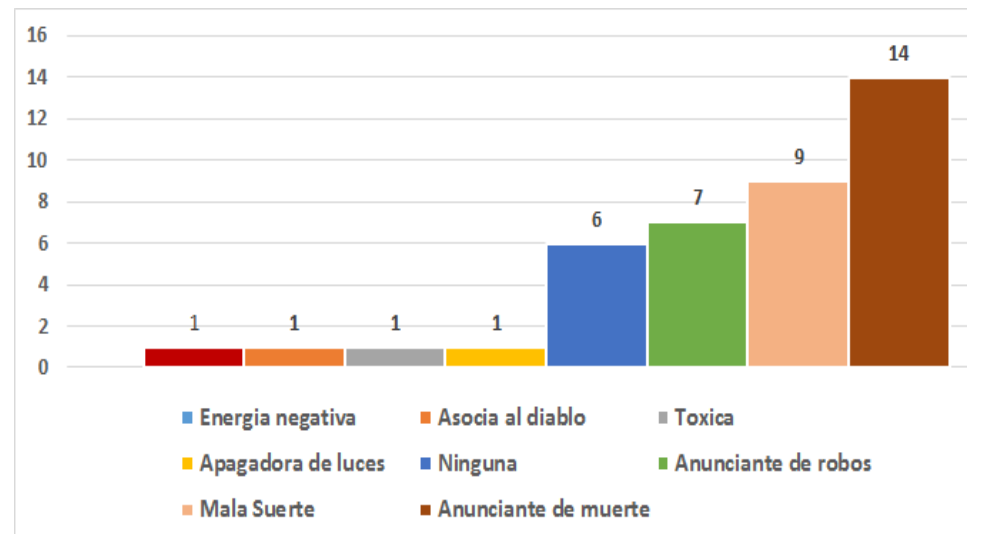

Figura 5: Significados asociado a la especie $A$. odorata por transeúntes de la Plaza Grande del Centro de Quito, Ecuador.

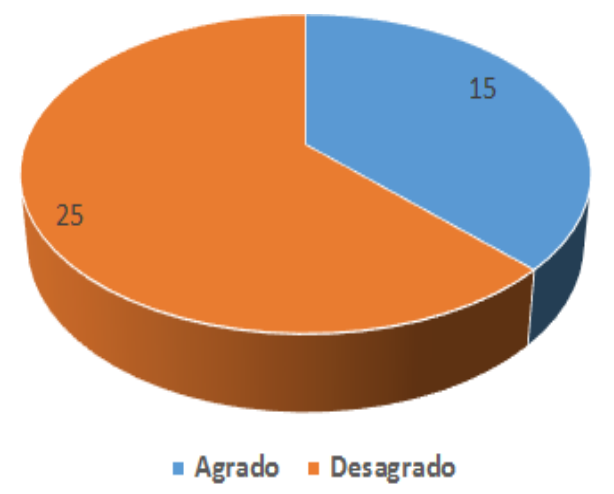

Figura 6: Sensación que se produce al observar a A. odorata. por transeúntes de la Plaza Grande del Centro de Quito, Ecuador.

El 56\% (14) de personas a quienes le desagrada la presencia de la mariposa en sus hogares establecieron que era debido a la apariencia del cuerpo y el 16\% (4) detalló que la presencia de pelos genera temor, sin embargo, la apariencia, textura, color de las alas y vientre no generaron temor en las personas (Fig. 7).

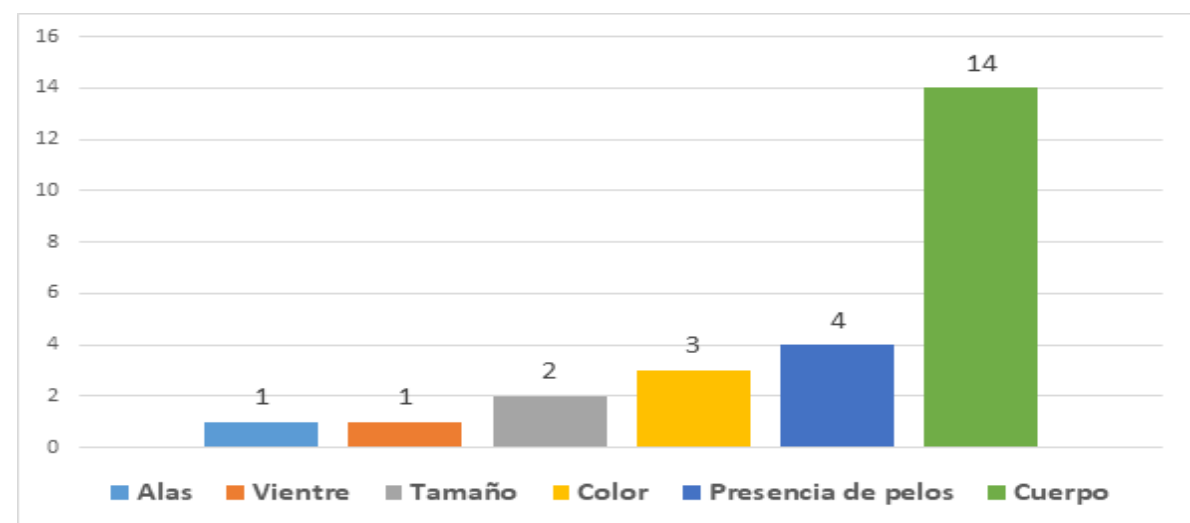

Figura 7: Razones de desagrado a A odorata por transeúntes de la Plaza Grande, Quito, Ecuador. 
En cuanto a la opinión de los informantes acerca del cambio a través del tiempo en la abundancia de la especie estudiada (Fig. 8), el 80\% (32) de los entrevistados afirmaron que, efectivamente, la abundancia de la mariposa nocturna ya no es la misma que hace 25 años.

Además, los entrevistados afirmaron que los cambios se deben al crecimiento poblacional de la ciudad, la aplicación de químicos en las casas, nuevas formas de control de plagas en las periferias de la ciudad, cambio climático y la superstición de ser mal agüero.

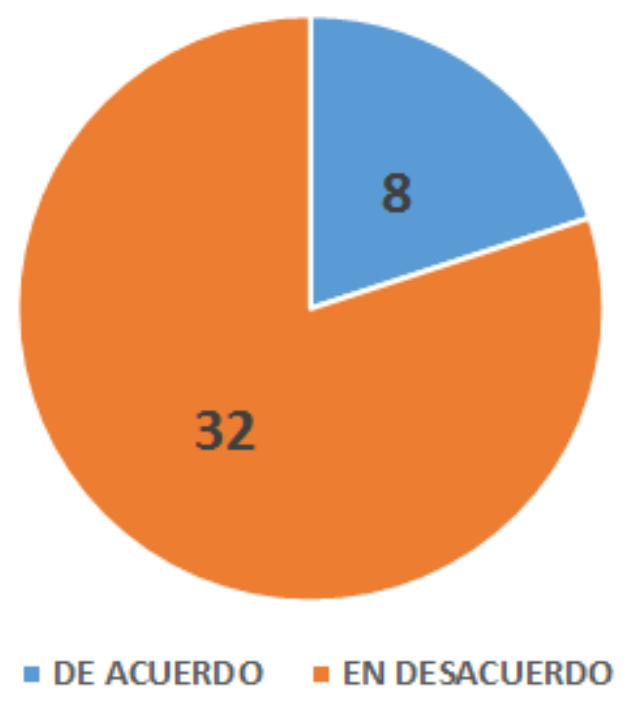

Figura 8: Opinión de los transeúntes de la Plaza Grande acerca del cambio en la abundancia de A. odorata.

El 35\% de entrevistados (14) afirmaron que la reacción frente a la especie consiste en matarlas, comúnmente mediante el uso de periódicos y matamoscas, mientras que un número reducido afirmó quemarlas vivas. El otro 35\% afirmó ahuyentarlas debido a que existe la posibilidad de que se cumplan los presagios mencionados anteriormente. El 27,5\% de los entrevistados (11) tiende a ignorar la presencia de la mariposa ya sea en sus casas o en espacios abiertos, mientras que el 2,5\% (1) afirmó sentirse intimidado o asustado por su presencia, sin recurrir a alguna acción violenta (Fig. 9). 


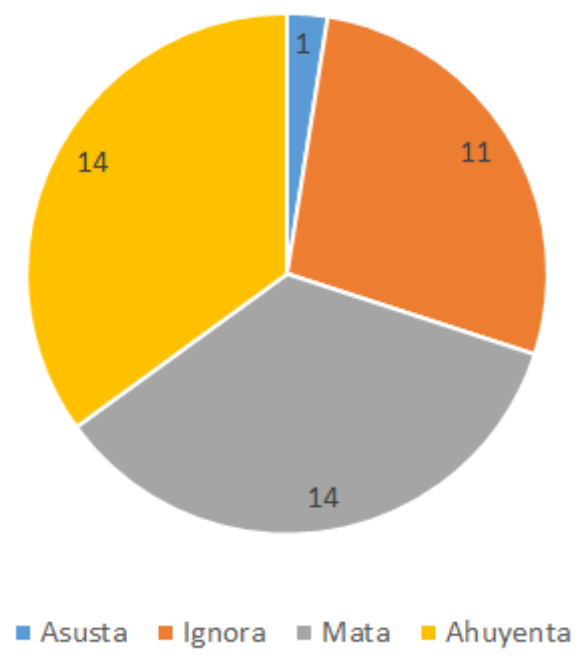

Figura 9: Reacción de las personas entrevistadas ante la presencia de A. odorata.

Finalmente, el conocimiento de las personas sobre la función en la naturaleza de $A$. odorata, el 50\% de los entrevistados (20) indicaron que esta especie no cumple ninguna actividad o función ecológica, seguida por un $15 \%$ (6) que mencionan que el periodo de nacimiento de esta mariposa es comúnmente en las épocas con abundantes lluvias o tiempos invernales. El 12,5\% (5) indicaran que sus hábitos alimenticios se basan principalmente en el consumo de néctar, además del 5\% (2) que apuntaran ser un importante organismo involucrado en la polinización. En cuanto al 17.5\% restante, mencionaron que esta especie presenta hábitos nocturnos, su crecimiento y desarrollo se da en sitios húmedos o en llanos, se alimentan de insectos y finalmente su hábitat es en las zonas rurales, con un porcentaje equivalente al $2,5 \%$ cada uno.

\section{DISCUSIÓN}

El nombre más común para Ascalapha odorata fue "Tandacuchi" seguido por “Mariposa de la muerte”. Según Barragán y colaboradores (2009) mencionan que estos nombres junto a la denominación "machaca" fueron usados desde la época precolombina del Ecuador. De la misma forma, se ha detallado que en países nórdicos es conocida como "mariposa bruja" o "mariposa maldita" (EKREM et al., 2014). Los nombres con significados negativos no se limitan únicamente al continente americano o europeo ya que en Madagascar la especie Erebus walkeri se la denomina como "espíritu de la muerte" (VAN HUIS, 2019).

El mayor porcentaje de los encuestados mencionaron que A. odorata es una mariposa nocturna "anunciante de la muerte" coincidente con las percepciones de ciertas 
comunidades Mesoamericanas, en las que la llegada de estas mariposas coincide con la época de celebración del día de los difuntos, en la que cada mariposa representa un familiar o difunto conocido (PÉREZ, 2011). Además, Barragán y colaboradores (2009) indican que cuando estas mariposas se posan dentro del hogar es porque una persona está próxima a morir lo cual concuerda con los criterios del significado de "anunciante de la muerte". Cabe mencionar que, en África, el estudio realizado por Van Huis (2019) sobre la importancia cultural de lepidópteros en 27 países de África Subsahariana determinó que los entrevistados mantienen una percepción negativa respecto al grupo de mariposas nocturnas debido a que se les asocia con muerte, enfermedades o eventos negativos. Lo anterior, las coloca en una posición difícil para incentivar su conservación, sin embargo, si bien las percepciones no se pueden cambiar fácilmente, su desmitificación puede alcanzar tolerancia o simpatía hacia los invertebrados (LOCKWOOD, 2013). El entorno social y cultural en el que se desenvuelve un individuo influye en su percepción del entorno por el conocimiento y experiencia que adquiere (LÓPEZ et al., 2018).

Por otra parte, el segundo aspecto de desagrado de los encuestados corresponde a sus setas o pelos urticantes, similar a lo mencionado por Van Huis (2019) quien sugiere que los pelos irritantes de las mariposas nocturnas han incidido directamente en la percepción negativa de las comunidades africanas.

Los entrevistados además indicaron que la abundancia poblacional de esta mariposa en la actualidad ha disminuido en comparación de hace 25 años, lo que puede estar relacionado a los cambios de temperatura y humedad generados por el crecimiento poblacional y la subsecuente urbanización en la ciudad de Quito (MONTERO et al., 2013). Así mismo, Leandro y Robert (2019) aluden la existencia de una "crisis de insectos" en razón a los desafíos que conlleva la conservación de invertebrados. Si bien se han establecido programas de conservación para especies silvestres, estos son enfocados para ciertas especies carismáticas lo que supone un reto en la conservación de otras especies por la falta de conocimiento.

Otra de las razones que afecta la salud de las poblaciones de estas mariposas radica en el uso de agroquímicos. Actualmente los pesticidas han sido los precursores de la disminución de varias poblaciones de insectos provocando efectos ecológicos irreparables (DEVINE et al., 2008). El ejemplo emblema es la disminución poblacional de Apis mellifera y otras especies de abejas nativas con un descenso de hasta $45 \%$ en Estados Unidos, un dato alarmante ya que son las principales polinizadoras dentro de los bosques aledaños a monocultivos (MARTIN; ARENAS, 2018). En general, los agroquímicos 
contaminan los hábitats naturales elevando los niveles de toxicidad y provocando envenenamiento en especies de insectos debido a su estrecho rango de tolerancia a factores físico-químicos (WASIM et al., 2009).

Con respecto al conocimiento sobre la función ecológica de esta especie, el $50 \%$ de los encuestados desconocen su importancia. Pese a esto, actualmente se conoce que las mariposas nocturnas son bioindicadores de la salud de un ecosistema, por lo cual es posible determinar la calidad que presentan los diversos hábitats (CARVAJAL; RIVERA, 2016). Además, son indicadores de estacionalidad, lo cual concuerda con los resultados obtenidos en este estudio, donde el $15 \%$ de los entrevistados afirmaron que en días lluviosos existe mayor avistamiento de esta especie, lo que se corrobora por el estudio realizado por Martínez (2018) quien indica que este aumento se debe a que en la estacionalidad fría aumenta la disponibilidad de recursos para hacer uso del follaje y al consumo de frutas como alimento. Así mismo, es necesario resaltar la importancia de A. odorata en la polinización, pues acorde al estudio elaborado por MacGregor (2019) las mariposas nocturnas son grandes polinizadores al igual que las abejas e incluso pueden complementar su trabajo viajando grandes distancias, lo cual previene la endogamia en especies vegetales, siendo la familia Noctuidae la más eficiente polinizadora (ORTEGÓN, 2017).

Finalmente, una de las actividades de divulgación científica fue la presentación de los resultados a manera de infografías durante el Ciclo de Conferencias de la Facultad de Ciencias Biológicas de la Universidad Central del Ecuador en el mes de agosto del año 2019.

\section{CONCLUSIONES}

Con los resultados obtenidos en este estudio se ha determinado que existe temor y una gran superstición en cuanto a la aparición de A. odorata. Es importante señalar que el conocimiento de los adultos mayores acerca de la ecología de esta especie es escaso. Su asociación a situaciones negativas ha dejado de lado la apreciación de su función ecológica, lo que ha conllevado como práctica común matarlas o ahuyentarlas debido a supersticiones.

El desagrado que origina esta especie es intrínseco a su existencia y a su naturaleza, pues su apariencia física es lo que causa repulsión en gran parte de los entrevistados, por lo que es necesario emprender y fortalecer proyectos de educación ambiental enfocados en la conservación de lepidópteros que a su vez permitan cambiar la percepción negativa que se les atribuye. 


\section{AGRADECIMIENTOS}

El presente trabajo investigativo fue realizado con la participación y previo consentimiento informado de adultos mayores transeúntes del Centro Histórico de la Ciudad de Quito. Además, queremos extender nuestro agradecimiento al Dr. Iván Vinicio Jácome - Negrete, quién nos ha incentivado a investigar la fauna de nuestro país desde el punto de vista etnobiológico.

\section{REFERÊNCIAS BIBLIOGRÁFICAS}

BARRAGÁN, A., DANGLES, O., CÁRDENAS, R. et al. The History of Entomology of Ecuador. Anales de entomología, v.45, n.4, p.410-423, 2009.

BERTONATTI, C. El taparaco o la mariposa de la muerte. Vida silvestre, v.125, n.1, 2013.

CARVAJAL, V; RIVERA, P. Las Fantásticas y desconocidas polinizadoras de la noche. Escuela Politécnica Nacional. Boletín Informativo del departamento de Biología, n.10, 2016, p.3-4.

CUEVA, S. Espacio público y patrimonio. Políticas de recuperación en el centro histórico de Quito. 1 ed. Quito: Ediciones Abya-Yala, 2010, 160 p.

CUVI, N. Las ciudades como mosaicos bioculturales: el caso del Centro Histórico de Quito. Revista Etnobiología, v.15, n.1, p.5-25, 2017.

DEVINE, G.; EZA, D.; OGUZUKU, E. et al. Uso de insecticidas: contexto y consecuencias ecológicas. Revista Peruana Médica de Salud Pública, v.25, n.1, p.74-100, 2008.

EKREM, T.; PETER, K.; WOODCOCK, T. et al. The Most Northerly Black Witch (Ascalapha odorata): A Tropical Moth in the Canadian Arctic. The Canadian Field Naturalist, v.60, p.77-79, 2014.

GARCÍA, A.; MOJICA, S.; BARRETO, S. et al. Estudio etnozoológico de las aves y mamíferos silvestres asociados a huertos frutícolas de Zacualpan de Amilpas, Morelos, México. Revista de Ciencias Ambientales, v.51, n.2, p.110-132, 2017.

LEANDRO, C.; ROBERT, P. Perceptions and representations of animal diversity: where did the insects go? Biological Conservation, v.237, p.400-408, 2019.

LOCKWOOD, J. The Infested Mind: Why Humans Fear, Loathe, and Love Insects. Oxford University Press. 2013. 203 p.

LÓPEZ, J. Entomofauna de importancia cultural en una comunidad maya-tzeltal de Chiapas, México. Estudios de la cultura Maya, v.50, 2017, p. 183-291.

LÓPEZ, E.; RUÍZ, L.; GÓMEZ, B. et al. Conocimiento y percepción tsotsil sobre los insectos perjudiciales para la milpa en la reserva de la selva El Ocote (Chiapas, México). Revista de 
Estudios de Cultura Maya, v.52, p.255-290, 2018.

MARTIN, N.; ARENAS, N. Daño colateral en abejas por la exposición a pesticidas de uso agrícola. Entramado, v.14, n.1, p.232-240, 2018.

MARTÍNEZ, C.; VÁZQUEZ, M. Mariposas y polillas fugaces en vuelos de la ciudad. Revista especies. México, v.8, n.32, p.4-5, 2006.

MARTÍNEZ, G. Enfermedad y entidades anímicas del entorno natural. Etiologías religiosorituales y espacio-ambientales entre los tobas del chaco central, argentina. Revista de Antropología Iberoamericana, v.5, n 2, p.189-221, 2010.

MARTÍNEZ, A. Dinámica temporal de la riqueza de especies y la abundancia de mariposas frugívoras (Lepidoptera: Nymphalidae) en la sabana. Revista Académica Colombiana Científica, v.42, n.164, p.246-254, 2018.

MACGREGOR, C.; KITSON, J.; FOX, R. et al. Construction, validation, and application of nocturnal pollen transport networks in an agro-ecosystem: a comparison using light microscopy and DNA metabarcoding. Ecological entomology, v.44, n.1, p.17-29, 2019.

MONTERO, J.; POZO, C.; CEPEDA, F. Recambio temporal de especies de Lepidópteros nocturnos en función de la temperatura y la humedad en una zona de selva caducifolia en Yucatán, México. Acta Zoológica Mexicana, v.29, n.3, p.614-628, 2013.

ORTEGÓN, L. Polillas, polinizadoras nocturnas que abundan en la Amazonia. Revista de la Agencia de Noticias de la Universidad Nacional de Colombia, Boletín de Prensa n.922, 2017.

PÉREZ, F. La celebración del día de muertos y su relación con la migración de las mariposas en Santa Cruz Nundaco, Ñuu Ndaco. Estudios Mesoamericanos, v.2, n.11, p.93-94, 2011.

SETH I.; PROCTOR, J.; ZEIGER, J. Juvenile Tyto alba furcata (Barn Owl) and Remains of Ascalapha odorata (Black Witch Moth) Found in Natural Limestone Cavity in Hellshire Hills, Jamaica. Caribbean Naturalist, v.37, p.1-11, 2017.

QUINN, M. The Black Witch (Ascalapha odorata) is the largest moth, if not the largest insect, north of Mexico. Texas Entomology, v.1, n.1, p.1-5, 2017.

RAMÍREZ, M. La Plaza Grande de Quito, fotografía y memoria. Series Magister, v.204, n.12, p.1-92, 2016.

VAN HUIS, A. Cultural significance of Lepidoptera in sub-Saharan Africa. Journal of Ethnobiology and Ethnomedicine, v.15, n.26, 2019, p.6-13.

VEGA, G. La interesante mariposa de la lotería Ascalapha odorata. Museo Nacional de Costa Rica. Editorial InBio, v. 365, 2013, p.1.

WASIM, M.; SENGUPTA, D.; CHOWDHURY, A. Impacto del uso de plaguicidas en la agricultura: sus beneficios y peligros. Interdisciplinary toxicology, v.2, p.1, p.1-12, 2009.

WAWRZYK, A. Saberes etnozoológicos de los pastores andinos: su importancia para la conservación y manejo de la vicuña (Vicugna vicugna). Ecología Austral, v. 23, p.156-164, 
BUITRÓN-BUITRÓN et al. Tandacuchis (Ascalopha odorata Linnaeus, 1978. Familia Noctuidai): percepciones míticas y sociales en el centro histórico de Quito. Ethnoscientia, 6, 2021. D.O.I.: 10.22276/ethnoscientia.v6i1.336 2013.

WILLIAMS, P. Polilla Bruja Negra. Revisión de Nashville, v.5, n.1, p.1, 2011. 